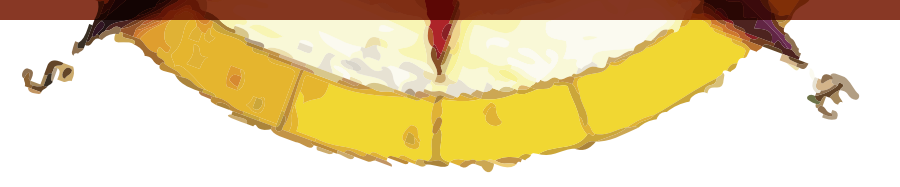

\title{
EL TERRITORIO LOCAL COMO RECURSO PARA EL APRENDIZAJE DE LAS CIENCIAS: UNA PROPUESTA DIDÁCTICA DESDE LA EDUCACIÓN PARA EL DESARROLLO SUSTENTABLE. RESULTADOS PRELIMINARES DE UNA INVESTIGACIÓN UNIVERSIDAD ESCUELA. ${ }^{1}$
}

\author{
Andoni Arenas Martija ${ }^{2}$ \\ Claudia Bruno Lagomarsino ${ }^{3}$ \\ Rodrigo Figueroa Sterquel ${ }^{4}$ \\ Carlos Pallacán Manque
}

\section{RESUMEN}

La baja calidad de los resultados de aprendizaje en ciencias, puede ser atribuido a una enseñanza descontextualizada, que no vincula los contenidos curriculares al entorno social y físico del estudiante ni de la escuela. A su vez, una falta de comprensión del propio territorio local por parte de los estudiantes y de los docentes, dificulta la generación de responsabilidad para con el medio ambiente y como consecuencia el no contar con futuros ciudadanos que sean actores activos de un Desarro-

$1 \quad$ El proyecto de investigación "El Territorio Local como Recurso para el Aprendizaje de las Ciencias: Una Propuesta Didáctica desde la Educación para el Desarrollo Sustentable" ha sido financiado por el Fondo Capital Semilla 2011 de la Vicerectoría de Investigación y Estudios Avanzados de la Pontificia Universidad Católica de Valparaíso, cuya ejecución está prevista para los años 2011-12.El Equipo de investigación Universidad-Escuela está formado por los docentes universitarios: Corina González (Biología), Germán Ahumada (Física), Cristian Merino (Química), Andoni Arenas (Geografía); los tesistas Gabriel Ramos, Betsabé Torres, Carlos Pallacán, Valentina Figueroa, Emilia Méndez, Camila Vicencio, Daniela Ibaceta, Paulina Bravo; los estudiantes Christopher Black y Sebastian Godoy de la PUCV, y por los profesores del Liceo José Cortez Braun de Viña del Mar, Ana María Soriano, Loreto Vergara, Marcelo Sánchez, Pedro Valdés, Ricardo Pérez, Leonardo Villaroel, Carlos Jeria, Oscar Carriel, Carmen Castillo y Janet Vilches. El equipo es coordinado por el profesor Víctor Salinas. De manera particular, el presente artículo pone énfasis en los hallazgos que vinculan los aspectos educativos geográficos, provenientes del proyecto como de la tesis de grado de Carlos Pallacán: "La enseñanza de la educación geográfica escolar y su vinculación al territorio local".

2 Profesor, Instituto de Geografía, Pontificia Universidad Católica de Valparaíso. Miembro Laboratorio de Didáctica de la Geografía y Desarrollo Profesional - GEOEDUCA

3 Profesora, Instituto de Geografía, Pontificia Universidad Católica de Valparaíso. Miembro Laboratorio de Didáctica de la Geografía y Desarrollo Profesional - GEOEDUCA

4 Profesor, Instituto de Geografía, Pontificia Universidad Católica de Valparaíso. Miembro Laboratorio de Didáctica de la Geografía y Desarrollo Profesional - GEOEDUCA

Miembro Laboratorio de Didáctica de la Geografía y Desarrollo Profesional - GEOEDUCA. 
1lo Sustentable para sus localidades y el país. El ojetivo de este proyecto es promover la inclusión de la Territorialidad como temática multidisciplinaria en el currículo escolar desde el enfoque de Educación para el Desarrollo Sustentable. A través del trabajo con los docentes que enseñan Biología, Química, Física Historia y Geografía en los niveles $1^{\circ}$ y $3^{\circ}$ de Enseñanza Media del Liceo José Cortés Brown, ${ }^{1}$ localizado en la comuna de Viña del Mar.

Palabras Clave: Territorio local, georeferenciación, mapeo curricular, secuencias didácticas, trabajo docente multidisciplinario, educación geográfica.

\section{RESUMO}

A baixa qualidade dos resultados da aprendizagem em ciências, pode ser atribuído a um ensino descontextualizado, o que não vincula os conteúdos curriculares ao entorno social e físico do estudante nem da escola. Por sua vez, uma falta de compreensão do próprio território local por parte dos estudantes e dos docentes, dificulta a geração de responsabilidade para com o meio ambiente e como consequência ou não contar com futuros cidadãos que sejam atores ativos de um Desenvolvimento Sustentável para suas localidades e seu país. O objetivo deste projeto é promover a inclusão da Territorialidade como temática multidisciplinar no currículo escolar desde o enfoque da Educação para o Desenvolvimento Sustentável. Através do trabalho com os docentes que ensinam Biologia, Química, Física História e Geografia em os níveis $1^{\circ}$ e $3^{\circ}$ de Ensino Meio do Liceo José Cortés Brown, ${ }^{2}$ localizado na comuna de Viña do Mar.

Palavras Chave: Território local, georeferenciação, mapeio curricular, sequências didáticas, trabalho docente multidisciplinar, educação geográfica.

\section{ABSTRACT}

The low quality in science learning results, can be explained by a de-contextualized teaching, that does not link curricular contents to social and student or school physical environment. In the same way, a low level of students and teachers understanding of their own local territory difficult responsibility with environment, and in the same way difficult the possibility to have in the future, citizens who are active players in sustainable development for their communities and countries. The principal idea of this project is to promote the territoriality inclusion as multidisciplinary thematic in the school curriculum, since sustainable development education, through the work made by biology, chemistry, physics, history and geography teachers in high school, levels $1^{\circ}$ and $3^{\circ}$ at José Cortés Brown School, in Viña del Mar.

Keywords: Local territory, georeferencing, mapping curriculum, teaching sequences, multidisciplinary teaching work, geographic education.

1 http://www.liceojcb.cl (3/8/2012).

2 http://www.liceojcb.cl (3/8/2012). 


\section{INTRODUCCIÓN}

A nivel nacional existe una baja calidad de los resultados de aprendizaje en ciencias, lo que entre otros factores, puede ser atribuido a una enseñanza descontextualizada, que no vincula los contenidos curriculares al entorno social y físico del estudiante ni de la escuela, lo que implica que a los estudiantes muchas veces no les haga sentido lo que están estudiando. A su vez, una falta de comprensión del propio territorio local por parte de los estudiantes y de los docentes, dificulta la generación de responsabilidad para con el medio ambiente y como consecuencia el no contar con futuros ciudadanos que sean actores activos de un Desarrollo Sustentable para sus localidades y el país. Los avances que se comentan se enmarcan en un proyecto que tiene como objetivos promover la inclusión de la Territorialidad como temática multidisciplinaria en el currículo escolar desde el enfoque de Educación para el Desarrollo Sustentable. A través del trabajo con los docentes que enseñan Biología, Química, Física Historia y Geografía en los niveles $1^{\circ}$ y $3^{\circ}$ de Enseñanza Media del Liceo José Cortés Brown, ${ }^{3}$ localizado en la comuna de Viña del Mar, se ha trabajado en establecer y potenciar espacios curriculares para el relevamiento del Territorio como un recurso estratégico en la enseñanza de estas disciplinas u otras disciplinas escolares. Del mismo modo, se espera incorporar a la práctica de los docentes metodologías adecuadas que permitan el uso del Territorio Local para la formulación de problemáticas relevantes para los estudiantes, de modo de promover en ellos no sólo el logro de las metas curriculares, sino también una comprensión de su entorno a través de la exploración y una actitud de respeto hacia el medio ambiente natural y humano.

En términos formales los objetivos del proyecto quedaron formulados de la siguiente manera, general: Promover la inclusión de la Territorialidad como temática multidisciplinaria en el currículo escolar desde el enfoque de Educación para el Desarrollo Sustentable estableciendo espacios curriculares para su relevamiento y metodologías adecuadas para su desarrollo en el aula de educación básica y media; específicos: 1) Realizar un mapeo del Currículo Nacional en el área de las Ciencias y la Geografía, considerando los niveles de $8^{\circ}$ a $3^{\circ}$ Medio, que permita identificar posibles componentes que permitan vincular con el concepto de Territorio Local desde un enfoque de la Educación para el Desarrollo Sustentable; 2) Realizar un catastro georeferenciado de los elementos del territorio local de establecimientos escolares con potencialidad de uso educativo; 3) Elaborar una propuesta formativa docente basada en la reflexión colaborativa a partir de la implementación del enfoque de la Educación para el Desarrollo Sustentable desde las potencialidades territoriales y a través de la construcción conjunta de secuencias didácticas; 4) Evaluar el impacto del proceso de formación docente en el aprendizaje de los estudiantes, en pos de la construcción de una nueva propuesta de perfeccionamiento.

\section{MARCO REFERENCIAL}

En la actualidad, la Ciencia constituye un eje estratégico del desarrollo humano, ya que implica no sólo el fortalecimiento de la capacidad crítica de una sociedad, sino también, una contribución a la inclusión y equidad social, bajo la idea de que en la actualidad el contar con competencias científicas - entendida como un conjunto de conocimientos, capacidades y actitudes científicas- permite una mejor comprensión del medio natural-social, de las realidades que allí se establecen y el poder actuar de manera participativa y fundamentada en el territorio. En Chile, los resultados de prue-

http://www.liceojcb.cl (3/8/2012). 
bas internacionales en Ciencia nos ubican por debajo del promedio internacional, y por sobretodo evidencian una alta inequidad en los aprendizajes. Lo anterior es crítico, toda vez que la falta de comprensión de contenidos científicos y su implicancia en la vida cotidiana redundan, también en una falta de comprensión del entorno por parte de los estudiantes, lo que a su vez dificulta la generación de responsabilidad para con el medio ambiente, y como consecuencia el contar con futuros ciudadanos que sean actores de un Desarrollo Sustentable del país.

Una causa probable que explica la baja calidad de los resultados de aprendizaje en ciencias, es una enseñanza que contextualiza poco los contenidos curriculares y no los vincula al entorno del estudiante ni al de la escuela, lo que implica que a los estudiantes muchas veces no les haga sentido lo que están estudiando. En el mejor de los casos, el entorno se menciona como un simple ejemplo de algún tópico, sin medios activos de pensamiento y acción, en vez de utilizarlo como recurso para problematizar situaciones relevantes al estudiante que sirvan para la real comprensión del territorio en que vive.

En general, esta falta de conexión entre el contenido a enseñar y el entorno del estudiante se expresa no sólo en la enseñanza que realiza el profesor en el aula, sino también en la relativa ausencia y/o baja relevancia de proyectos curriculares en enseñanza de las Ciencia que valoren el entorno natural y cultural en que estudian y viven los estudiantes, entendido este como Territorio Local. Por otra parte, y observando el currículo escolar chileno, existe una cierta dispersión curricular, que dificulta el uso del territorio y su comprensión de manera integral así como de muchos conceptos integradores. A menudo, incluso dentro de una misma asignatura, las unidades aparecen desconectadas entre sí, dificultando una visión integral y más compleja de lo que es la naturaleza y las sociedades. Menos frecuente aún es la presentación integrada de contenidos curriculares de diferentes asignaturas, lo cual incide en que en general, no existe un trabajo colaborativo multidisciplinar por parte de los docentes y de modo general no se incentiva a ello.

De esta manera, y si bien hay una intención de inter y multidisciplinariedad desde los Contenidos Mínimos Obligatorios y los Objetivos Fundamentales (evidenciable en particular en los Mapas de Progreso $)^{1}$, se requieren enfoques y materiales que planteen de manera más explícita y concreta esta integración. Por otra parte, los propios textos escolares, como la mayoría de los materiales didácticos son genéricos para todo el país, y no contextualizan a la realidad de cada situación en particular, lo que no fomenta el uso didáctica del Territorio Local por parte del docente ni la comprensión de él por parte de los estudiantes. Bajo este escenario, se hace fundamental involucrar a los docentes en trabajos de reflexión colaborativa que les permitan incorporar a sus prácticas el uso del Territorio desde una visión multidisciplinaria donde la Geografía ocupa un lugar preponderante.

\section{METODOLOGÍA}

Considerando que se ha tenido como objetivo que los docentes que participantes de esta propuesta tuvieran la posibilidad de reflexionar sobre un cambio en sus prácticas pedagógicas en vistas a la mejora en los aprendizajes de sus estudiantes, que involucraran al Territorio como recurso en su

$1 \quad$ El actual Gobierno de Chile y sus autoridades educacionales han decidido reformular los Mapas de Progreso Existente. Los vigentes se pueden consultar en http://www.saladeprofes.com/se-dice/586-mapas-de-progresode-mineduc.html (3/8/2012) y los ya reformulado para la Educación Parvularia en http://www.mineduc.cl/ index5 int.php?id portal $=47 \&$ id contenido $=17116 \&$ id seccion $=3264 \& \mathrm{c}=362(3 / 8 / 2012)$. 
enseñanza, lo que, a su vez, se expresará en el desarrollo de ciertos conocimientos, habilidades y actitudes en los estudiantes orientadas a la comprensión del Territorio en que habitan. Es por ello que, para lograr estas condiciones, se aplicaron una serie de estrategias metodológicas que buscan promover la inclusión-discusión de la Territorialidad como temática multidisciplinaria. A continuación se presentan tres de las estrategias metodológicas implementadas: Construcción de los conceptos claves, Elaboración de un Mapa Curricular y Georeferenciación de elementos del Territorio Local.

\section{a. Construcción de un concepto clave: Territorio}

Para la definición de los conceptos claves no se partió de una idea previa y cerrada. La alternativa fue la construcción colaborativa de los conceptos claves. A continuación se ejemplifica con el procedimiento para la formulación del concepto de Territorio.

1.- Inicio de la reunión, tesistas, profesores del liceo y profesores de la universidad: información y aspectos administrativos.

2. Definición personal de Territorio (5 líneas).

3. Agrupación por disciplinas. Discusión definiciones personales y consenso 5 conceptos comunes de lo que define al Territorio, esto a partir de las definiciones personales.

4. Plenario (grupos disciplinarios comparten definiciones y conclusiones). Uso notas autoadhesivas (con los conceptos propuestos). Cada grupo explica sus conceptos, las notas son reordenadas. Discusión decantando elementos a considerar o no en la conceptualización de territorio.

5. Contrastación con definición formal de Territorio aportada por el equipo PUCV (Ver Figura $\mathrm{N}^{\mathrm{o}}$ 1). Síntesis de los elementos señalados por la definición formal en la lista de conceptos propuestos en la reflexión colectiva.

6. Durante la reunión se toman notas de campo, fotografías y video grabación, sobre todo de la parte de la contrastación.

7. Después de la reunión, con los insumos recogidos se sistematiza en una imagen la conceptualización trabajada-acordada a partir de la nube de palabras que entrega el sitio web Wordle (http://www.wordle.net). Semejante procedimiento se usa para los conceptos de Desarrollo Sustentable y Secuencias Didácticas.

\section{b. Elaboración de un Mapa Curricular}

Se entiende como mapa curricular a la representación gráfica que se hace a una o varias disciplinas curriculares que permiten la distinción de elementos y la organización de los contenidos que la componen. Siguiendo a Azúa (2007), este concepto es sinónimo de plan o programa, ya que muestra la estructura general de una asignatura, un curso o una carrera, por lo que de manera sintética se puede visualizar la distribución y organización los contenidos que se relacionen con un determinado tema, en este caso, la disciplina geográfica, tomando como soporte uno o varios programas de estudio que componen el currículo de formación escolar. 


\section{Conceptualización Formal de Territorio.}

De modo general se puede señalar que el Territorio es el espacio geográfico adscrito a un ser, a una comunidad, a un ente de cualquier naturaleza, física o inmaterial: el espacio de vida de una especie animal, el área de aparición de una comunidad vegetal, el ámbito de difusión de una lengua o de cualquier otra práctica social como una creencia o la implantación de una tecnología.

Cuando se atribuye a un grupo humano complejo (un pueblo, una nación, una sociedad) se convierte en uno de los elementos constitutivos, fundamentales de su proyecto común: en soporte y recurso básico, ámbito de vida, con un paisaje propio e invariantes en la memoria personal y colectiva. En definitiva en el espacio geográfico en el que se vive y que corresponde manejar y administrar para bien de los individuos y del conjunto de la comunidad (Zoido, 1998).

De esta manera el Territorio es el Espacio Geográfico apropiado, puesto en valor y en el que se advierten las condiciones de un ejercicio efectivo del poder político, en el sentido de poder transformador de la sociedad y la naturaleza que posee el género humano. Alude a un espacio efectivamente usado, tasado o en reserva, resumen de las relaciones históricas entre la sociedad y la naturaleza (Moraes y Da Costa, 1993 en Gurevich 2005).

El Territorio es multidimensional, proyectivo, dinámico, construido socialmente y representado. El territorio es multidimensional, característica que queda explicitada en la siguiente afirmación: es el espacio de asentamiento de una comunidad, el hábitat de una población, y encierra también sus dimensiones sociales, económicas y culturales. Se ve afectado por las actividades socio-económicas que la sociedad desarrolla en el mismo, considerando tanto su ocupación física, como el ejercicio político sobre el mismo y el derecho a su uso.

En términos temporales el Territorio es también una proyección, lo que significa que debe ser comprendido en términos de presente-futuro. El territorio no es sólo lo que "es" actualmente, sino también lo que "va siendo". De esta manera, además de conocer los recursos - naturales y humanos - de un territorio, y su situación desde el punto de vista ambiental, se requiere conocer las expectativas, la percepción, las imágenes y los proyectos que la sociedad ha depositado en él, considerando tanto el sector privado como el público, los actores individuales como los colectivos.

El territorio es dinámico: El territorio es más que el soporte físico en donde se desarrolla una comunidad. Es también el resultado, el reflejo, la manifestación de la cultura de un grupo social, que lo va transformando progresivamente y éste a su vez, modifica a sus habitantes. Existe una dinámica de interrelación entre el territorio y sus habitantes, modificándose mutuamente. Es decir, el Territorio también es "lo que puede ser"1. El territorio es construido socialmente: no existe el territorio "per se". Un territorio cobra interés y tiene una valoración de diversa índole, en función del grupo social y cultural que lo analice, de los niveles tecnológicos disponibles, de las diversas ideologías imperantes, así como de las circunstancias históricas que les toca vivir ${ }^{2}$.

El territorio es un espacio apropiado colectivamente a través de representaciones, mentales y físicas, y por lo tanto conociendo éstas últimas es que podremos ordenarlo en función de criterios preestablecidos.

Este aspecto implica por otra parte que el territorio de la población puede ser diferente del territorio jurídico o administrativo de un estado o país: las dos visiones no siempre coinciden. En el caso de Chile, los territorios de las regiones, provincias y comunas, como entidades de administración no se superponen directamente con las identidades culturales asociadas a esos territorios jurídicos.

Referencias Bibliográficas:

- Cátedra Evaluación Recursos Territoriales, Carrera de Geografía, Instituto de Geografía, Pontificia Universidad Católica de Valparaíso. 2010

- GUREVICH, R. (2005). Sociedades y territorios contemporáneos. Una introducción a la enseñanza de la geografía. Fundo de la Cultura Económica. Buenos Aires, Argentina.

- MINEDUC, (2010). Objetivos Fundamentales y Contenidos Mínimos Obligatorios de la Educación Básica y Media. Actualización 2009.

○ Moraesy Da Costa, (1993). A valorizaçao do espaço. Hucitec. Sao Paulo.

- ZOIDO, F. (1998). Didáctica de las ciencias sociales. Geografia e Historia, Barcelona: No 16. Nuevas fronteras de los contenidos geográficos, pp. 19-31. Disponible en la web: http://www.ub.edu/geocrit/orden.htm

Para Profundizar:

Gustavo MONTAÑEZ GÓMEZ y Ovidio DELGADO MAHECH (1998) ESPACIO, TERRITORIO Y REGION: CONCEPTOS BASICOS PARA UN PROYECTO NACIONAL. CUADERNOS DE GEOGRAFÍA. Vol.VI1 No. 1-2 1998. Disponible en la web:

www.geolatinam.com/files/Montanez y Delgado._1998.pdf

Figura $\mathrm{N}^{\mathrm{o}}$ 1: Conceptualización de Territorio presentada como definición formal para la reflexión colectiva. Fuente: Documento preparado para la discusión por Carlos Pallacán y Andoni Arenas. Instituto de Geografía-PUCV. 
La importancia de estas herramientas se valora en la perspectiva de poderse orientar o ubicar - de ahí la analogía con el mapa - en el currículum escolar por medio de la identificación de elementos, secuencias en un temática o en determinados sectores de aprendizaje y las relaciones que se puedan generar entre ellos.

Para el desarrollo transversal de un determinado tema a considerar es necesario preguntarse: ¿qué contenidos se espera que integren los y las estudiantes?, ¿qué habilidades se espera que desarrollen?, ¿qué valores se espera que vivencien?, ¿en qué sectores y niveles se encuentran esos contenidos? Y ¿cuáles son las actividades a través de las cuales se espera desarrollar las habilidades y valores? (MINEDUC, 2007), por tanto, los Mapas Curriculares brindan un soporte fundamental a la hora de empezar a develar dichas interrogantes.

Su funcionalidad se liga a la información que se produce respecto a los aprendizajes que se pueden esperar de los estudiantes y a partir de esta base, realizar acciones que se complementen por medio de otros espacios o mecanismos que permitan la formación y el desarrollo curricular. De igual modo su utilidad puede ser ofrecida a las escuelas para entregar una visión general de los programas y partir de este primer acercamiento, desarrollar materiales pertinentes a una determinada disciplina y sus distintos niveles de aprendizaje.

Teniendo en consideración el marco del proyecto de investigación y el estudio de caso dondeLiceo José Cortés Brown- se procedió al desarrollo de diversas actividades con el de recabar información sobre la temática central de este estudio, ligado a la importancia del territorio local en el proceso de aprendizaje de la educación geográfica escolar. Para ello se realizó un barrido en cuanto los aspectos geográfica en el subsector de aprendizaje Historia, Geografía y Ciencias Sociales. Para ello se recurre a la herramienta del Mapeo Curricular, con el cual se compararon y analizaron los distintos instrumentos formativos como lo son los Objetivos Fundamentales, Objetivos Fundamentales Transversales, Contenidos Mínimos Obligatorios y Mapas de Progreso del Aprendizaje, sumado a la conceptualización del territorio, respecto a la incidencia que poseen los contenidos en la disciplina geográfica y su posible vinculación con el entorno local, con el propósito de generar herramientas didácticas que permitan a los estudiantes estrechar los contenidos que se les enseñan con su contexto inmediato, pudiendo generar análisis e interrelaciones entre los diversos factores y elementos que intervienen en su diario vivir, así como ayudar a sus docentes a facilitarles este proceso educativo.

\section{c. Catastro Georreferenciado}

Una de las inquietudes que mayormente compartían los docentes del establecimiento al momento de iniciar el proyecto y hacer el nexo entre los estudiantes y el Territorio Local, era tener una aproximación respecto a los sectores de la comuna en donde vivían los estudiantes, visto desde una mirada espacial. Es por ello que se solicitó como producto, la generación de un catastro georreferenciado llevado a soportes cartográficos, en donde se pudiera localizar los hogares de los estudiantes correspondientes a los niveles en los cuales trabajaría el proyecto, correspondientes a $1^{\circ}$ y $3^{\circ}$ de Enseñanza Media.

La información entregada por la Unidad Técnica Pedagógica del establecimiento fue espacializada por medio del Sistema de Información Geográfica ArcGis 9.3, complementándola con la información entregada por el SECPLA de la Ilustre Municipalidad de Viña del Mar correspon- 
diente a los sectores censales de la comuna. Es así como por medio de la herramienta SpatialJoin de ArcGis se le asignó a cada polígono correspondiente a los sectores de la comuna, la cantidad de estudiantes que poseía cada uno. Posterior a ello, la cantidad de estudiantes por sector se jerarquizó en 3 categorías: Baja - Media - Alta, utilizando Quiebres Naturales (Natural Breaks), puesto que esta clasificación expresa de manera proporcional la distribución de áreas homogéneas en una misma unidad territorial.

\section{d. Cuestionario de Percepción y Espacialización de Resultados.}

Con el objetivo de conocer la visión de los estudiantes respecto al vínculo que puedan tener con el territorio local, se elaboró un Cuestionario de Percepción que buscaba recopilar la información necesaria para el posterior trabajo con el desarrollo de las secuencias didácticas, de manera que éstas involucraran aquellos elementos y relaciones que los propios estudiantes perciben ocurrir en su entorno local.

El cuestionario contaba con 2 ítems, el primero ligado a la Pertenencia a un Lugar, destacando elementos tales como:

- Sectores de Proveniencia

- Tipo de Locomoción Utilizada en el trayecto hacia el Liceo

- Tiempo de Viaje en el trayecto de ida hacia el Liceo

- Elementos de la ciudad significativos en el trayecto hacia y desde el Liceo

- Tipo de actividades extracurriculares realizadas tanto en la comuna como en algún sector en particular.

- Sectores de la ciudad en donde exista un mayor nivel de identificación

Mientras que el segundo ítem consistía en propuestas para desarrollar en clases que le parecieran interesantes a los estudiantes para desarrollar tanto dentro de la comuna, como de la ciudad de Valparaíso u otros espacios regionales. Estas propuestas debían ir ligadas a los diferentes subsectores en los cuales tiene implicancia el proyecto: Matemática, Arte, Filosofía, Física, Química, Biología, Historia, Geografía y Ciencias Sociales.

Finalmente es necesario destacar que este instrumento fue aplicado a los niveles $1^{\circ}$ y $3^{\circ}$ de Enseñanza Media, puesto que tales niveles son impartidos por la totalidad de los docentes que participan de éste proyecto.

Para este caso, se presentaran los resultados del ítem Elementos de la ciudad significativos en el trayecto hacia y desde el Liceo, el cual consistía principalmente en espacializar aquellos elementos o sectores de la comuna de Viña del Mar que mayor interacción o vínculo tenían con los estudiantes del liceo. Para este caso, todos aquellos elementos identificados se clasificaron según la tipología que realiza Lynch (1984): Senda, Bordes, Barrios, Nodos, Hitos. Finalmente se generaron polos dentro de la ciudad los cuales poseían una mayor concentración de estos elementos mencionados 
anteriormente.

\section{RESULTADOS}

\section{a. Territorio: Concepto Clave}

La primera parte de la construcción colaborativa del concepto de territorio significó la agrupación-jerarquización de las palabras principales usadas para denominarlo a través de la técnica de "nube de palabras" (wordcloud) presentadas en la Figura $\mathrm{N}^{\mathrm{o}} 2$.

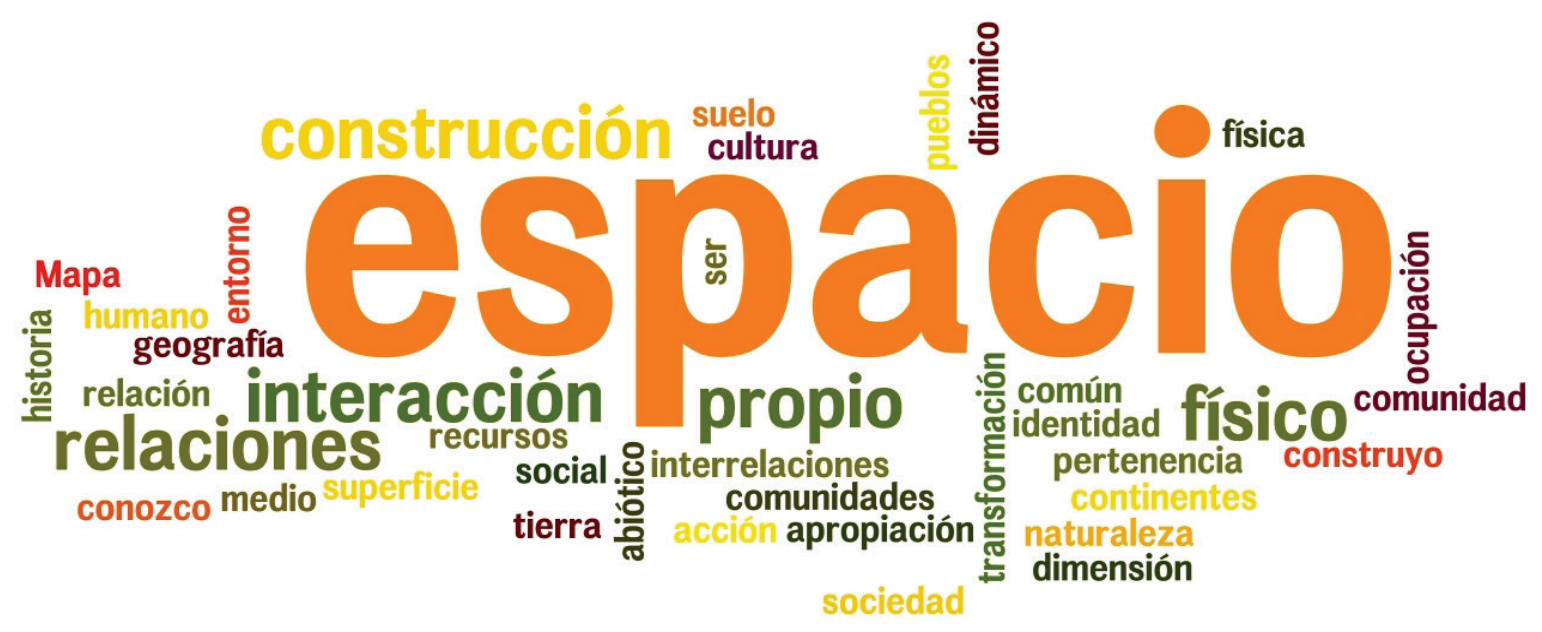

Figura $\mathrm{N}^{\mathrm{o}} 2$ : Nube de palabra generada de acuerdo a la conceptualización de territorio por el equipo de investigación. Fuente: Pallacán, 2012 Con la aplicación Wordle.

La discusión en torno a este agrupamiento-jerarquización presentó diversas preguntas acerca del significado del concepto: ¿debemos incorporar al hombre-mujer en la definición?, ¿se puede imaginar el concepto de territorio sin el espacio físico? Por lo general se entiende que el concepto siempre está vinculado con un alguien, quien siente como suyo el territorio generando un espacio propio, lo que está en relación con el arraigo que el cerro, escuela u hogar genera; una "topofilia".

Se puede entender como una construcción social que depende de una determinada percepción. De esta forma, el concepto es multidimensional, incorporando múltiples perspectivas que dependen de diferentes comunidades. La pertenencia sería la construcción emocional del territorio, el cual es percibido de forma subjetiva. Por ejemplo, la forma de entender el liceo José Cortés Brown de Cerro Castillo (Viña del Mar) y como se hace la diferencia "con los de Recreo" (otro barrio cercano en Viña del Mar). Por otro lado, la relación de los estudiantes con su entorno y los servicios que allí existen.

El territorio no solo es el suelo y el hombre-mujer sino que es la relación. La acción entre ellos y 
su espacio, lo que incorpora elementos simbólicos y lo que se construye en el espacio en sí, como por ejemplo la infraestructura. En este sentido, el concepto es dinámico: "modificamos el territorio y el territorio le hace llevar una forma de vida".

\section{b. Mapa Curricular potencial de interdisciplinariedad}

De acuerdo a lo establecido por el MINEDUC, se consideran los Objetivos Fundamentales y los Contenidos Mínimos Obligatorios como la orientación principal para la elaboración de programas de estudio, que serán el punto de partida para la planificación de clases (MINEDUC, 2009, p. 199).

De este modo, la sistematización del Mapeo Curricular toma los dos instrumentos mencionados anteriormente como ejes principales en la localización de aspectos en común, para posteriormente generar la relación con los Objetivos Fundamentales Transversales y el Mapa de Progreso en su dimensión Espacio Geográfico. Una vez establecida las relaciones entre los cuatro instrumentos, se procede a instalar la vinculación que tales contenidos poseen con el territorio local, permitiendo a los estudiantes poder comprender los fenómenos espaciales y poseer un manejo de conocimiento a nivel multiescalar.

Es así como el nivel primero de enseñanza media se configura en seis campos de estudio, cinco de los cuales tienen por objetivo entender al siglo XX como el período en donde se encuentran las raíces de los logros, problemas y conflictos de la sociedad actual, estos corresponden a:

Las Guerras Mundiales y el Nuevo Orden Político Internacional.

El Mundo en la segunda mitad del s. XX

Los procesos de urbanización del s. XX

Geografía de la Población Mundial

El Mundo Globalizado con sus logros y falencias.

Mientras que el sexto corresponde a un campo más práctico orientado al desarrollo de habilidades por parte del estudiante para poder indagar, interpretar y emitir opiniones respecto a la sociedad contemporánea.

A modo de ejemplo se presenta el primer campo de estudio, ligado a Las Guerras Mundiales y el Nuevo Orden Político Internacional.

Para este campo se tomaron como aspecto en común el primer ítem de lo Objetivos Fundamentales en conjunto con las dos primeras unidades que describe los Contenidos Mínimos Obligatorios. Como se puede apreciar en la Figura $\mathrm{N}^{\circ} 3$, para el caso del Mapa de Progreso de Aprendizaje se relacionó con el ítem perteneciente al nivel 4 que da cuenta de cómo los seres humanos a través del tiempo se adaptan y transforman el espacio geográfico, mientras que los Objetivos Fundamentales para éste campo no vinculan ni relacionan ningún ámbito respecto a los Objetivos Fundamentales Transversales. 


\begin{tabular}{|c|c|c|c|}
\hline \multicolumn{4}{|c|}{ INSTRUMENTOS CURRICULARES HISTORIA, GEOGRAFIAY CIENCIAS SOCIALES } \\
\hline \multicolumn{4}{|c|}{$\underline{1^{\circ} \text { AÑO ENSEÑANZA MEDIA }}$} \\
\hline$\frac{\text { OB JETIVOS }}{\text { FUNDAMENTALES }}$ & $\begin{array}{r}\frac{\text { OBJETIVOS }}{\text { FUNDAMENTAL- }} \\
\frac{\text { ES TRANSVER- }}{\underline{\text { SALES }}}\end{array}$ & $\frac{\frac{\text { CONTENIDOS }}{\text { MÍNIMOS OBLIGA- }}}{\underline{\text { TORIOS }}}$ & $\begin{array}{r}\text { MAPA DE PRO- } \\
\text { GRESO NIVEL 4-5 }\end{array}$ \\
\hline $\begin{array}{l}\frac{\text { Caracterizar las prin- }}{\text { cipales dimensiones }} \\
\frac{\frac{\text { del proceso de global- }}{\text { ización y sus }}}{\text { consecuencias espacia- }} \\
\frac{\text { les, económicas y cul- }}{\text { turales. }}\end{array}$ & 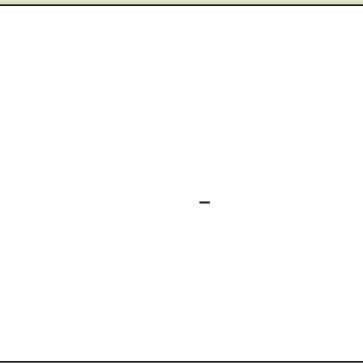 & $\frac{\frac{\text { El mundo global- }}{\text { izado con sus logros y }}}{\underline{\text { falencias }}}$ & $\begin{array}{c}\frac{\text { Comprende que a }}{\text { través del tiempo }} \\
\underline{\text { han cambiado las formas }} \\
\text { en que los seres humanos } \\
\underline{\text { se adaptan y transforman }} \\
\underline{\text { el espacio geográfico. }}\end{array}$ \\
\hline
\end{tabular}

Figura No 3: Sistematización Mapeo Curricular en el campo Guerras Mundiales y el nuevo orden político internacional. Fuente: Pallacán, 2012 base a MINEDUC, 2009.

Cabe destacar que el ejercicio de Mapeo Curricular realizado por el equipo participante del proyecto para el subsector de Historia, Geografía y Ciencias Sociales fue estableciendo ejes temáticos para ser incluidos desde una escala local. Es por ello que para éste campo, se vinculó el eje Procesos Migratorios y las repercusiones que trajo tal fenómeno en las comunas de Viña del Mar y Valparaíso fenómeno, así como los patrones de localización de la población migrante y su influencia en el desarrollo político, económico, arquitectónico y cultural de las comunas anteriormente señaladas. La sistematización de éste campo se ve expresado por medio de la Figura N²

\section{c. Catastro Georreferenciado}

Una vez realizado el proceso de georreferenciación, se pudo determinar que de un total de 107 alumnos que componen el $1^{\circ}$ año de enseñanza media, 86 habitan en la comuna de Viña del Mar, destacando principalmente a los sectores de Achupallas y Forestal, quienes captan alrededor de un $40 \%$ del total del nivel comunal, otorgándoles una categoría Alta, tal como se puede apreciar en la Figura No5.

Como particularidad, es posible apreciar que el nivel analizado presenta una homogeneidad en cuanto a la distribución de los alumnos de acuerdo a la orientación Norte-Sur, puesto que el sector de Forestal se encuentra en el sur de la comuna, mientras que Achupallas se emplaza en el sector norte.

Sumando al sector de Achupallas, se destaca al sector norte de Viña del Mar como quien otorga mayor cantidad de alumnos al Liceo con una cifra del $45 \%$ del total de los sectores de la comuna, considerando que Gómez Carreño, Reñaca Alto y Santa Inés poseen una cantidad total de 23 alumnos, cifra que corresponde a un $80 \%$ de un determinado curso. 


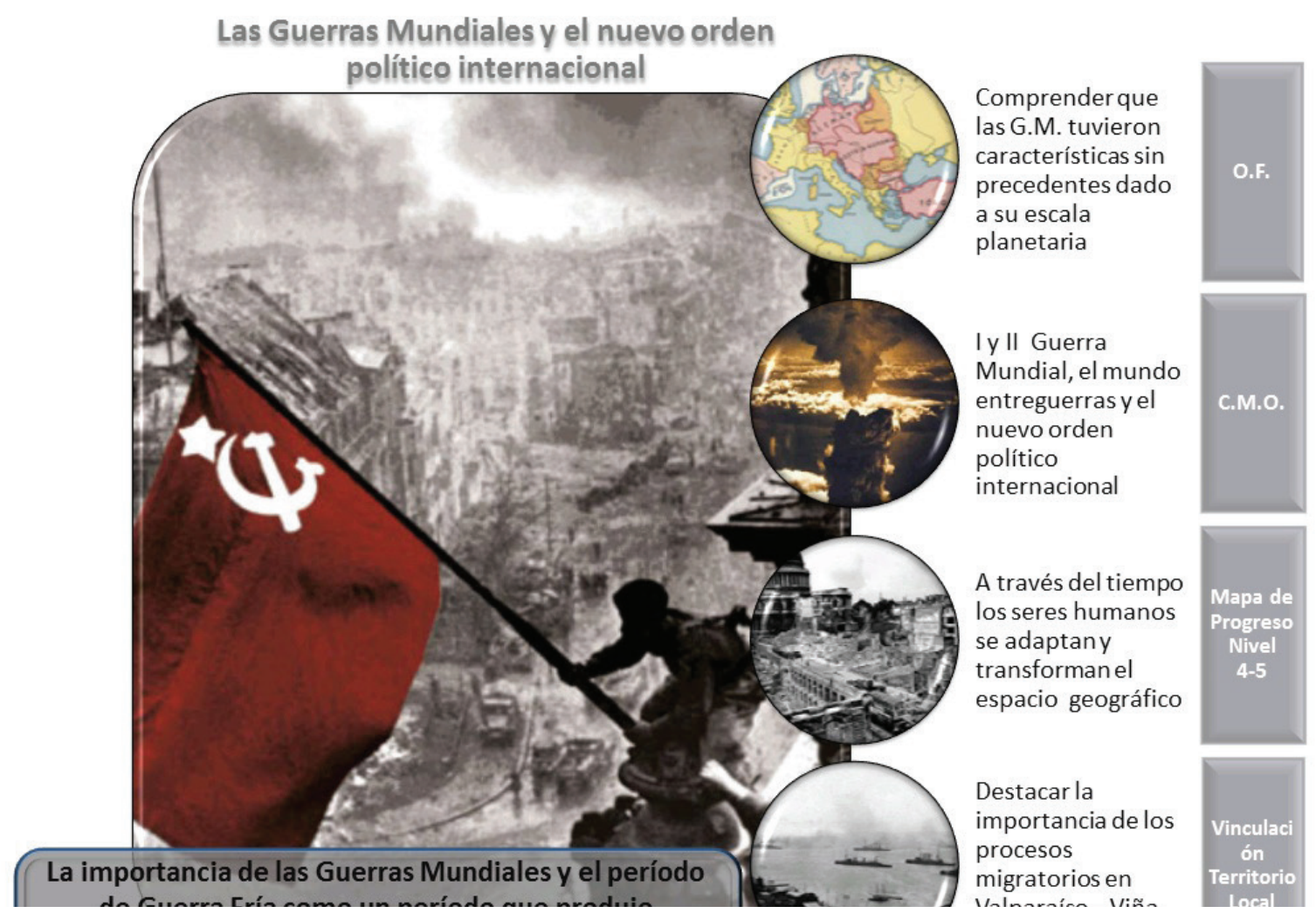

Figura $\mathrm{N}^{\mathrm{o}}$ 4: Sistematización Mapeo Curricular en el campo Guerras Mundiales y el nuevo orden político internacional. Fuente: Pallacán, 2012.

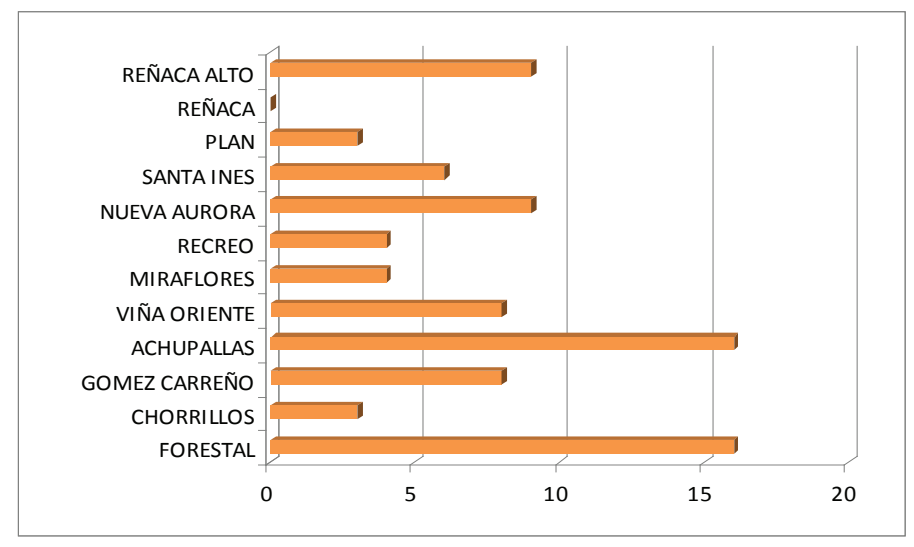

Figura $\mathrm{N}^{\circ}$ 5: Cantidad de alumnos de acuerdo a sector de Viña del Mar. Fuente: Pallacán, 2012. 


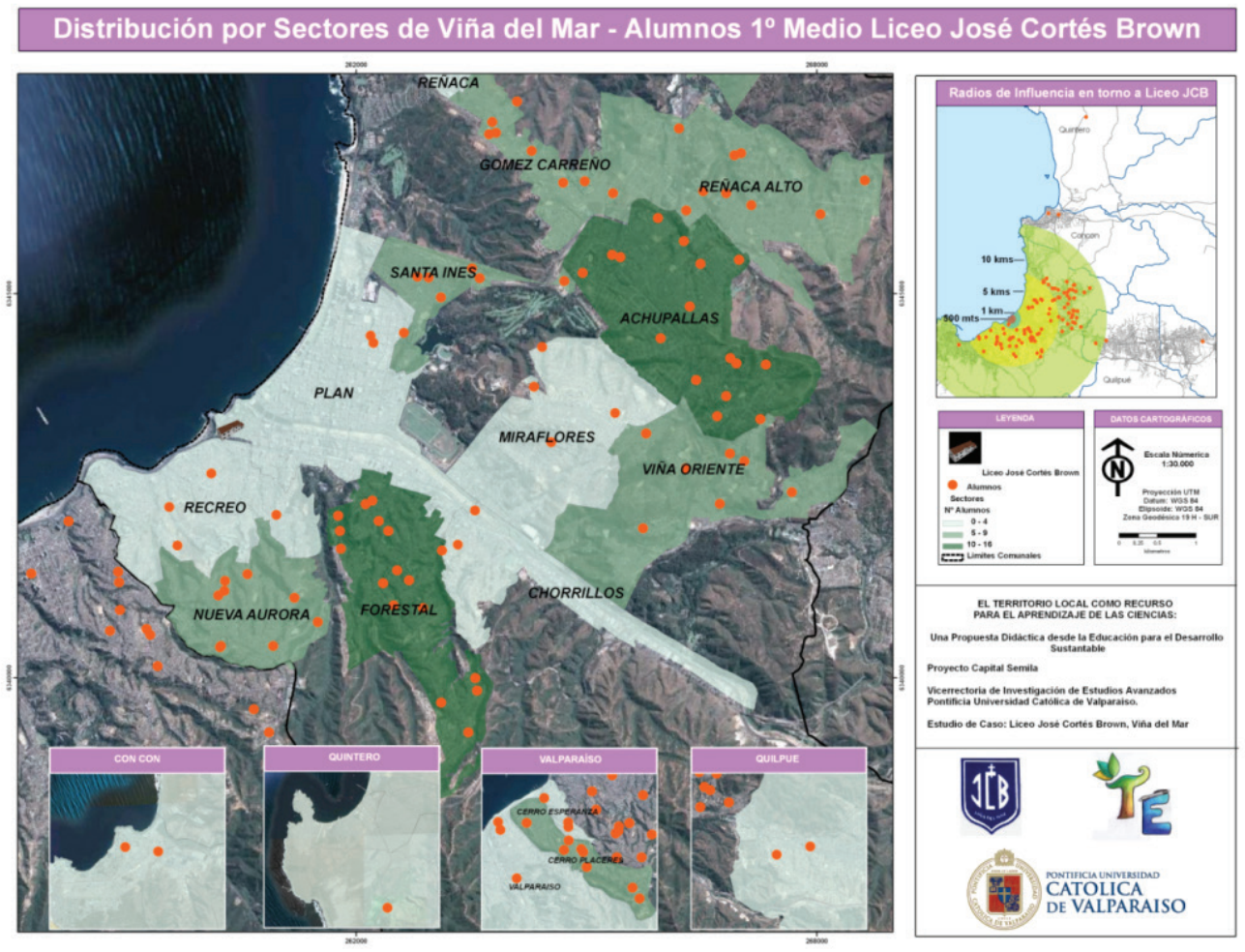

Figura $\mathrm{N}^{\mathrm{o}}$ 6: Espacialización de estudiantes se acuerdo a los sectores de proveniencia. Fuente: Pallacán, 2012.

Una singularidad que ocurre con el nivel analizado, es que sólo el $8 \%$ del total de los estudiantes que habitan en Viña del Mar, se encuentra en los sectores de mayor cercanía al liceo, como lo es el sector Plan de Viña y Recreo. En una situación similar, se encuentran los sectores de Miraflores y Chorrillos, quienes comprenden un 9\% del total comunal como lo muestra la Figura $\mathrm{N}^{\mathrm{o}} 6$.

En cuanto a los alumnos provenientes de otras comunas, estos corresponden a 21, de los cuales la mayoría proviene de Valparaíso, especialmente de los cerros más cercanos a Viña del Mar como lo son Esperanza y Placeres. El resto de los alumnos provienen de las comunas de Quilpué, Villa Alemana, Concón y Quintero.

\section{d. Cuestionario de Percepción y Espacialización de Resultados.}

En base al Cuestionario de Percepción en donde cada alumno debía mencionar 6 elementos del territorio local correspondiente a la comuna de Viña del Mar que más le llamaban su atención y/o realizaban algún tipo de interacción de acuerdo al trayecto ida - vuelta hacia el establecimiento y siguiendo la tipología de Lynch mencionada anteriormente, se analizó la información y fue dividida en 4 categorías. La categoría Bordes no se utilizó puesto que en el total de respuestas vinculadas a este ítem, no se encontraron elementos suficientes para considerarlos representativos.

De acuerdo a la Figura $N^{0}$ 7, se presentan a continuación aquellos elementos que tuvieron una 
mayor cantidad de reiteración por parte de los alumnos, según la categoría anteriormente mencionada:

\begin{tabular}{|c|c|c|c|}
\hline \multicolumn{4}{|c|}{ ELEMENTOS IDENTIFICADOS CON MAYOR GRADO DE REITERACION } \\
\hline$\underline{\text { Sendas }}$ & $\underline{\text { Barrios }}$ & $\underline{\underline{\text { Nodos }}}$ & $\underline{\underline{\text { Hitos }}}$ \\
\hline$\underline{\text { Calle 15 Norte }}$ & $\underline{\text { Castillo }}$ & $\underline{\text { Reloj de }}$ & $\underline{\text { Parroquia Nuestra Señora de }}$ \\
\hline$\underline{\text { Calle Quillota }}$ & - & - & $\underline{\text { Dolores }}$ \\
\hline$\underline{\text { Variante Agua }}$ & & - & $\underline{\text { Hospital Gustavo Fricke }}$ \\
\hline$\underline{\text { Santa }}$ & - & - & $\underline{\text { Liceo René Descartes }}$ \\
\hline$\underline{\text { Calle Arlegui }}$ & - & - & \\
\hline
\end{tabular}

Figura $N^{0}$ 7: Identificación de elementos con mayor grado de reiteración en la comuna de Viña del Mar. Fuente: Pallacán, 2012.

Para el caso de las sendas cabe destacar que la importancia de los ejes 15 Norte y Quillota radica principalmente por ser aquellas calles por las cuales circulan los recorridos de microbuses provenientes de los sectores norte de Viña del Mar tales como Gómez Carreño, Reñaca Alto o Achupalladas, mencionados anteriormente como aquellos que aportan una gran cantidad de alumnos al establecimiento. El caso de la calle Arlegui es principalmente destacado por ser el eje por el cual circulan los recorridos provenientes del sector Forestal, mientras que la variante Agua Santa es el eje articulador de los sectores correspondientes a Recreo y Nueva Aurora.

Por su parte Cerro Castillo se menciona como el barrio de la comuna con mayor reconocimiento, este hecho se atribuye principalmente a ser el barrio de confluencia de los alumnos desde todos los sectores de proveniencia, puesto que es acá donde se localiza el Liceo José Cortés Brown.

El Reloj de Flores es el elemento que mayor se destaca no sólo en la categoría nodos, sino que en todos los elementos de la ciudad como se muestra en la tabla anterior con un total de 47 reiteraciones. Esta circunvalación considerada un elemento clave por los alumnos se debe a que se encuentra el paradero de microbús del cual provienen los recorridos desde los sectores con mayor cantidad de encuestados, especialmente al sur del Liceo como también todos aquellos provenientes de Valparaíso

Finalmente los hitos destacan principalmente por encontrarse próximo a los sectores mayormente poblados por los estudiantes y ser centros de grandes aglomeraciones como hospitales, mall o supermercados. El caso del liceo René Descartes destaca por encontrarse próximo al establecimiento en cuestión.

Con el objetivo de generar una red entre todos los elementos mayormente reiterados en las categorías analizadas, se conjugaron en un solo soporte cartográfico, definiendo de tal modo los límites al interior de la comuna de Viña del Mar en los cuales existe mayor interacción por parte de los alumnos.

Es posible apreciar que la conformación de estos cuatro elementos más mencionados por los 
encuestados genera una red de carácter perpendicular, confluyendo en el centro de la ciudad, dentro del cual se localizan una nutrida cantidad de elementos con menor relevancia cercado por los ejes viales Arlegui por el norte y por Viana - Álvarez por el sur, y desde el hospital Gustavo Fricke por el oriente hasta llegar al extremo occidente correspondiente al Cerro Castillo, tal como se aprecia en la siguiente Figura $\mathrm{N}^{\circ} 9$.

Es así como a modo de síntesis es posible de identificar tres polos que estructuran el eje perpendicular señalado anteriormente; Polo 15 Norte, Polo Centro de Viña del Mar y polo Cerro Castillo, cada uno con elementos señalados bajo las categorías analizadas, los cuales se convierten en el soporte base para la utilización de dichos espacios al momento de realizar levantamiento de información por medio de actividades en terreno que se deseen programar los docentes del establecimiento, como a su vez integrarlos dichos territorios a los contenidos presentes en los instrumentos curriculares como forma de ejemplificar algún hecho, fenómeno o situación que quieran demostrar de manera tangible y cercana a los estudiantes.

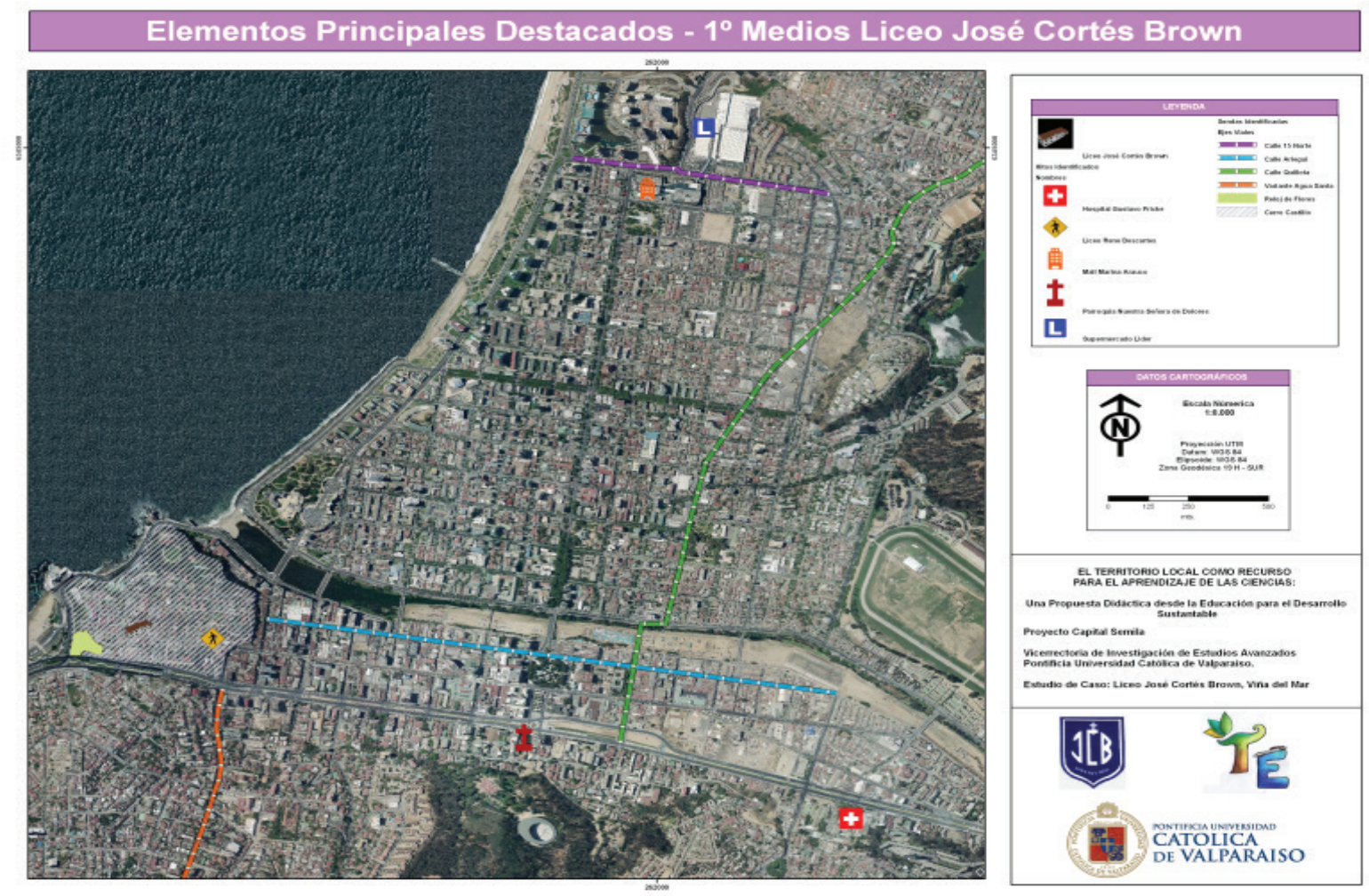

Figura $\mathrm{N}^{\circ}$ 9: Espacialización de elementos con mayor grado de reiteración por parte de los estudiantes. Fuente: Pallacán, 2012.

\section{CONCLUSIONES}

Teniendo en consideración que el proyecto aún está en ejecución, es preciso señalar que las conclusiones presentadas son provisorias, sobre todo en lo que respecta al aporte concreto al aprendizaje de los estudiantes que tendrán la aplicación de Secuencias Didácticas cuyas bases estén en el 
Territorio como recurso educativo fundamental. ${ }^{1}$

Sin embargo, con la elaboración del Mapeo Curricular por disciplinas-asignaturas, se logro homogeneizaron todos los instrumentos curriculares que tienen a disposición los docentes, con el propósito de interrelacionarlos bajo ciertas contenidos/habilidades considerados estructurantes para la formación de los estudiantes. En el caso del nivel $1^{\circ}$ de Enseñanza Media, para la asignatura de Historia, Geografía y Ciencias Sociales se trabajó en torno al eje: Guerras Mundiales, procesos migratorios y los efectos en la configuración del mundo actual. De este modo, el Mapa Curricular se convierte en el soporte base con el cual se implementará una secuencia didáctica bajo la perspectiva de que las temáticas planteadas por el eje forman parte del territorio local en que habitan los estudiantes y se localiza el liceo. De esta manera el Mapeo Curricular, como proceso y los Mapas Curriculares, como productos se convierte en claves para un trabajo docente de orden profesional e multidisciplinaria en vistas a la mejora en los aprendizajes de los estudiantes.

La profundidad del Mapeo Curricular y la calidad de sus productos (mapas) se deben en parte a la discusión y reflexión en torno al proyecto y sus objetivos, así como en los modos de trabajo. Un lugar central en ello fue la elaboración colaborativa de los significados para los conceptos centrales del proyecto, como se ha dicho más arriba, de Territorio, Secuencia Didáctica y Desarrollo Sustentable. Esta elaboración de significado colectiva permitió tener una base común en la que convergen las miradas disciplinarias y sus implicancias pedagógicas, validando una "redacción" en la que todos se sentían contribuyentes y representados, aunque no fuera total ni exclusiva para cada participante. Este punto de encuentro, es además, el "canal o puente" para la interdisciplinariedad, puesto que en el significado de Territorio, por ejemplo, se reconocía lo que las otras disciplina compartían, complementaban o podían apoyar a la enseñanza de la propia al trabajar con el territorio en que se circunscribe el liceo y los estudiantes. Además, en esto, se ratifica, que la mirada y las temáticas geográficas tienen amplio alcance y potencial multidisciplinaria, revalorizando una educación geográfica más amplia y profunda.

A ello se le suma la espacialización de los resultados del cuestionario que permitió sintetizar y categorizar los sectores de la comuna de Viña del Mar y fuera de ella, donde se localizan los hogares de los estudiantes y la identificar de los elementos de referencia presentes en la ciudad, constituyendo la oportunidad de visualizar, sobre todo para los profesores/as, el territorio vivido por los estudiantes, con la consiguiente mayor comprensión de la realidad de los estudiantes y del espacio en que ellos mismos habitan.

Es así como por medio de la construcción y aplicación de esta propuesta, ligada a instancias de participación e involucramiento de los propios docentes y a futuro, de los estudiantes, con su propio territorio geográfico en el cual realizan sus actividades cotidianas. A partir de todo esto se cree posible generar un aporte a la construcción de mejores aprendizajes y a una educación geográfica con sentido de pertinencia, desarrollando habilidades ligadas al análisis del espacio visto desde una perspectiva sistémica, tal como lo propone Souto (2011: 39), al señalar que "la necesidad de cambiar la forma tradicional de enseñar Geografia, de tal manera que los niños y adolescentes la entiendan y apliquen en su vida cotidiana, despertando el sentido crítico y la formación ciudadana". 


\section{BIBLIOGRAFÍA}

Acero, V. y Cabeza, I. (2008). ¿Educación y Territorio o Territorialidad Educativa? Una alternativa para acercarnos a la enseñanza de la geografía y las ciencias sociales. Universidad Pedagógica y Tecnológica de Colombia.

Albertini, R., G. Cárdenas-Jirón, J. Babel, G. Díaz Veliz, J. Ryzaguirre, A. Labra y R. Lewin (2005). Enseñanza de las ciencias a nivel escolar y formación en ciencia en el pregrado universitario. En: Ureta, T., J. Babul, S. Martínez y J. Allende. Análisis y Proyecciones de la Ciencia Chilena 2005. Academia de Ciencias. Recuperado el 30-01-12 de: http://www.academia-ciencias. $\underline{\mathrm{cl} / \text { ? module=investig. }}$.

Araya, F. La Didáctica de la Geografía en América Latina: Experiencias y Tendencias. Revista electrónica Geográfica Austral Año 2, No 1 enero-junio 2010. (12 págs.) [en línea] http:// revistanadir.yolasite.com/resources/Didactica.pdf [consulta: 27 septiembre de 2011]

Arenas, A.; Figueroa, R. (2010). El lugar de la Geografia en la enseñanza escolar. Reflexiones a propósito del Ajuste Curricular y el potencial de las Tecnologías de la Información y la Comunicación, TIC. Anales Sociedad Chilena Ciencias Geográficas 2009. 467 - 474 págs.

Audigier, F. (2002). Un estudio sobre la enseñanza de la historia, la geografia y la educación cívica en la escuela elemental de Francia: temas, métodos y preguntas. Enseñanza de las ciencias sociales, V.1, pp: 3-6.

Cely, A. Construcción Socio-Cultural de los Territorios Urbanos. Una Indagación desde la Educación. Revista Anekumene. (140 -155 págs.) Vol. 1, No 1, 2011. [en línea] http://www.anekumene. com/index.php/revista/article/view/15/16 [consulta: 21 de abril de 2012]

Chin, C.; Chia, L-G. (2004). Problem-basedlearning: Usingstudent's questions to drive knowlegde construction. Science education, V. 88, n. 5, pág. 707-727.

Garlick, R. ; Laugksch, R. C. (2008). Teaching children to ask investigable questions in science. School Science Review. Vol. 90, n. 331. Pág. 119-127.

Gurevich, R. (2005). Sociedades y territorios contemporáneos. Una introducción a la enseñanza de la geografía. Fondo de la Cultura Económica. Buenos Aires, Argentina.

Imbernon, F. (2004). La investigación educativa como herramienta de investigación del profesorado: reflexión y experiencias de investigación educativa. Barcelona, Graó.

Lee Y. C. (2007). From a museum demonstration to problem a solving: promoting the construction of concepts. Physics Education. V. 42, n. 4, pág. 378-384.

Lynch, K. (1984). La Imagen de la Ciudad. Barcelona, España: Editorial G. Gili. 228 páginas.

Macedo, C. y Salgado, C. (2007). Educación ambiental y educación para el desarrollo sostenible en América Latina. Forum Sostenibilidad: UNESCO. 
Mineduc, (2010). Objetivos Fundamentales y Contenidos Mínimos Obligatorios de la Educación Básica y Media. Actualización 2009.

Mineduc. (2009). Fundamentos del ajuste curricular para el sector de ciencias Naturales. Ministerio de Educación de Chile. Santiago de Chile.

Montañez G. y Delgado, O. (1998). Espacio, Territorio y Region: conceptos basicos para un proyecto nacional. Cuadernos de Geografía. Vol.VI1 No. 1-2 1998. Disponible en la web: www.geolatinam.com/files/Montanez y Delgado. 1998.pdf

Moraes y Da Costa (1993). A valorização do espaço. Hucitec. São Paulo.

Norton S.; Mcrobbie, C. y Ginns, I. 2007. Problem solving in a middle school robotics desing classroom. Reseach in Science Education. V. 37, n3, pp. 261-277.

Pallacán, C. (2012). La enseñanza de la educación geográfica escolar y su vinculación territorial: estudio de caso. Liceo José Cortés Brown, Viña del mar. Pontificia Universidad Católica de Valparaíso; Valparaíso, Región de Valparaíso; Chile. Tesis: Geógrafo. Profesor guía: Negrete Sepúlveda, Jorge Javier o Arenas Martija, Andoni Patricio.

Reigosa, C.; Jimenez-Aleixandre, M. (2007). Scaffoided problema-solving in the physics and chemistry laboratory: Difficulties hindering students assumption of responsibility. International Journal of Science Education. V. 29, n3, pp. 307-330.

Rivera, J. La cultura juvenil desde la enseñanza de la Geografia en el contexto del mundo contemporâneo. Revista Anekumene. (85 - 100 págs.) Vol. 1, No 1, 2011. [en línea] http://www.anekumene.com/ index.php/revista/article/view/11/12 [consulta: 21 de abril de 2012]

Souto, X. Una educación geográfica para el siglo XXI: Aprender competencias para ser ciudadano en el mundo global. Revista Anekumene. (28 - 47 págs.) Vol. 1, No 1, 2011. [en línea] http://www. anekumene.com/index.php/revista/article/view/8/9 [consulta: 21 de abril de 2012].

Svarzman, J. Enseñar Geografia en la escuela hoy. Revista Radio 12(ntes). (20 págs.). No 4, Año 1. 2009. [en línea] www.12ntes.com/wp-content/uploads/12ntes-digital-4.pdf [consulta: 07 septiembre de 2011]

Zarate, A. (1995). Aprendizaje significativo y geografia de las representaciones mentales. Anales de Geografía, Madrid: Universidad Complutense, pp. 931-840.

Zoido, F. (1998). Didáctica de las ciencias sociales. Geografía e Historia, Barcelona: No 16 . Nuevas fronteras de los contenidos geográficos, pp. 19-31. Disponible en la web: http://www.ub.edu/ geocrit/orden.htm.

\section{(Footnotes)}

$1 \quad$ Lo cual supone poder desarrollar capacidades para "comprender el propio territorio. Por ejemplo: "Las habilidades geográficas tienen como propósito desarrollar en alumnos y alumnas la ubicación espacial y el análisis territorial, en el cual han de poder integrar diversas variables para adquirir una visión dinámica y sistémica del espacio que habitan". 
MINEDUC, 2010, p. 197.

2 El Marco Curricular para el Sector de Historia, Geografía y Ciencias Sociales señala explícitamente: "Este sector también se propone que los estudiantes desarrollen el pensamiento espacial, con una visión dinámica y sistémica, que les permita comprender el territorio como una construcción humana posible de modificar en beneficio de la calidad de vida de las personas que lo habitan" (MINEDUC, 2010, p. 195).

Artículo recibido el 30 de mayo de 2012 y aprobado el 30 de junio de 2012. 